\title{
The impact of excessive maternal weight on the nutritional status of the fetus - the role of leptin
}

Lidia A. Biesiadaํㅡ, Ewa Głowackaํ, Michał Krekora², Stanisław Sobantka ${ }^{3}$, Aleksandra Krokocka², Grzegorz Krasomski²

\author{
${ }^{1}$ Center for Medical Diagnostic Laboratory, Polish Mother's Memorial Hospital \\ Institute, Lodz, Poland \\ 2Department of Obstetrics and Gynecology, Polish Mother's Memorial Hospital \\ Institute, Lodz, Poland \\ ${ }^{3}$ Second Department of Gynecology and Obstetrics, Medical University of Lodz, \\ Lodz, Poland
}

Submitted: 12 May 2014

Accepted: 12 August 2014

Arch Med Sci 2016; 12, 2: 394-401

DOI: 10.5114/aoms.2016.59267

Copyright (c) 2016 Termedia \& Banach

\section{Abstract}

Introduction: Assessment of leptin concentration in the blood of pregnant women (leptin concentration - Lc) and in their newborns, according to their nutritional status.

Material and methods: Pregnant women $(n=42)$ were divided into normal body mass (NBM) and excessive body mass (EBM) groups. Neonates were divided into AGA (eutrophic) and LGA (macrosomic) groups. Leptin concentraction was studied in 4 subgroups: NBM/AGA, NBM/LGA, EBM/AGA, EBM/LGA. Results: Mothers: A significant correlation was found between maternal LC and body mass index (BMI) $(r=0.75, p<0.001)$. Maternal LC was 3 times higher than neonatal Lc $(p<0.00001)$. The NBM mothers showed lower LC compared to EBM mothers $(p=0.000018)$. Leptin concentration values in NBM/LGA and NBM/AGA mothers were similar $(p=0.6775)$. Newborns: Correlations were found between LC and ponderal index $(\mathrm{PI})(r=0.67$, $p<0.001)$, weight $(r=0.43, p=0.004)$ and placental weight $(r=0.56$, $p<0.001)$. Girls presented higher Lc than boys ( $p=0.0338)$. In LGA groups, newborns born to EBM mothers presented higher Lc than those born to NBM mothers $(p=0.0013)$. In both AGA groups, Lc was similar (AGA/EBM vs. AGA/ $\operatorname{NBM} p=0.1619)$. The highest $L C$ and the largest placentas were found in the group of LGA newborns born to EBM mothers.

Conclusions: Leptin concentration positively correlates with $\mathrm{BMI}$ in pregnant women, with $\mathrm{PI}$ and female sex in newborns as well as with placental weight. The nutritional status of fetuses does not affect the LC of their nonobese mothers. The LGA neonates born to EBM women demonstrate higher LC than LGA neonates born to NBM mothers, whereas LC of AGA neonates is similar regardless of the nutritional status of their mothers.

Key words: leptin, maternal body mass index, large for gestational age newborn.

\section{Introduction}

Leptin is a polypeptide hormone encoded by the ob gene, which plays a key role in maintaining the energy balance. Leptin is one of the adipokines produced and secreted essentially by adipocytes of the white adipose tissue, and it is believed that its concentration in serum cor-

\author{
Corresponding author: \\ Lidia A. Biesiada PhD \\ Center for Medical \\ Diagnostic Laboratory \\ Polish Mother's Memorial \\ Hospital Institute \\ 281/289 Rzgowska St \\ 93-338 Lodz, Poland \\ Phone: +48 601976933 \\ Fax: +48 422711750 \\ E-mail: bieslidia@o2.pl
}


relates with body mass index (BMI) [1-3]. In the central nervous system leptin inhibits the activity of neurons expressing neuropeptide $Y$ and Agouti-related protein and stimulates neurons expressing pro-opiomelanocortin, which gives a satiety signal and leads to the reduction of appetite [4-6].

The physiological effect of leptin, however, is not limited to the regulation of appetite and body mass. There is currently great research interest in the role played by leptin in the regulation of peripheral tissue metabolism, angiogenesis, tissue maturation processes, immunomodulation and reproduction $[1,2,7,8]$. Leptin plays a central role in implantation, induces secretion of chorion gonadotropin in trophoblast cells, increases mitogenesis and regulates the development of the placenta, which is a very important source of it [1, 2, 6, 7, 9]. Furthermore, the leptin concentration in the maternal serum increases from the very early weeks of pregnancy, and it is present in the amniotic fluid and the cord blood. These findings suggest that leptin may play an important role in the physiological development of pregnancy.

Serum leptin concentration depends mainly on the amount of white adipose tissue. A high level of leptin is observed in patients suffering from all components of the metabolic syndrome. Other significant factors are inflammatory or neoplastic processes. Monajemzadeh found that leptin levels in patients with cystic fibrosis were higher than in controls and suggested that infants may have shown higher concentrations than adult patients [10].

In an uncomplicated pregnancy, fetal growth is dependent on genetic, placental and hormonal factors as well as the intrauterine environment, which is determined by maternal metabolic status and energy resources [7, 9-11]. Leptin, through its role in controlling energy stores, seems to be an important factor in fetal intrauterine growth. However, in contrast to the infant or adult, the fetus cannot refuse food intake, and the amount of incident nutrients is dependent on a combination of their concentration in the maternal blood and the placental transport efficiency. Therefore, the direct effect of leptin as the satiety factor in utero is as yet inconclusive [1].

Obesity is characterized by hyperleptinemia and leptin resistance [6]. In pregnant women, these disorders and the increased supply of dietary nutrients induce maternal hyperlipidemia and insulin resistance. As a result, increased amounts of glucose and free fatty acids (FFAs) become available for the fetus. These conditions can stimulate fetal excessive intrauterine growth, leading to the birth of large for gestational age (LGA) newborns, whose abundant adipose tissue is also a source of leptin.

There are believed to be three sources of leptin in pregnant women: maternal adipose tissue, fetal adipose tissue and placenta. The interrelationships between these departments have now been widely investigated $[2,6,8]$. Many studies address the leptin concentration in the blood of children born to women with gestational diabetes, preeclampsia or intrauterine fetal growth restriction (IUGR). However, little is known about this subject in obese pregnant women without other accompanying diseases. Because leptin is the main hormone responsible for maternal lipolysis in advanced pregnancy and its secretion in obese people is increased, the link between leptin levels and mothers' and newborns' body mass has been the subject of our work.

The purpose of this study is to investigate the following questions:

1. Is there a relationship between maternal leptin serum concentration and:

- newborn's leptin concentration?

- maternal BMI and age?

2. Does fetal nutritional status affects the mother's leptin concentration?

3. Is there a relationship between newborns' leptin levels and:

- their birth weight and Rohrer index (ponderal index-PI)?

- their sex?

4. Does the excessive maternal weight affect the newborns' leptin concentration?

\section{Material and methods}

This project was approved by the ethics committee of the Polish Mother's Memorial Hospital Institute. The aim and the manner in which the study would be performed were explained to each patient, and written consent was obtained from all women.

Forty-two healthy pregnant women aged from 17 to 43 and their children were examined. All women delivered above the $37^{\text {th }}$ week of gestation. The pre-pregnancy BMI was calculated for each mother, and the ponderal index (PI) that characterizes the degree of body slenderness was calculated for each newborn. The research was conducted on a group of healthy pregnant women who did not show any abnormalities in basic and biochemical blood tests before pregnancy. There was no ethnic diversity in the examined group. None of the recruited women had a history of hypertension, atherosclerosis, dyslipidemia, diabetes mellitus, coronary artery disease, cancer, chronic inflammatory diseases, liver or kidney diseases, autoimmune diseases, coagulopathy or genetically determined diseases (cystic fibrosis) before pregnancy.

Body composition of all patients was assessed just before delivery. The percentage of muscle mass, fat mass, and visceral fat mass were determined using the bioelectrical impedance method. 
Patients with edema, polyhydramnios or oligohydramnios were excluded from study due to the expected impact of these factors on the result of the body composition test. A measurement of the waist circumference was abandoned due to the advancement of pregnancy.

Patients with pregnancy complications, particularly gestational diabetes, pregnancy-induced hypertension and intrauterine growth retardation (IUGR) were excluded.

The nutritional status of each newborn was assessed by specifying the birth weight of the newborn in relation to its gestational age based on Polish population centile charts. Children were divided into two groups: appropriate for gestational age (AGA), whose weight was situated between the $10^{\text {th }}$ and $90^{\text {th }}$ percentiles, and large for gestational age (LGA), whose weight was situated above the $90^{\text {th }}$ percentile.

Mothers were divided into two groups according to their pre-pregnancy BMI. Women whose BMI ranged between 18.5 and $24.99 \mathrm{~kg} / \mathrm{m}^{2}$ were defined as normal body mass mothers (NBM, $n=20$ ). Women whose BMI was more than $25 \mathrm{~kg} / \mathrm{m}^{2}$ were called excessive body mass mothers (EBM, $n=22$ ).

Taking into account the nutritional status of mothers and newborns, four tested subgroups were separated:

- group 1 - normal body mass mothers who delivered appropriate for gestational age children (NBM/AGA); $n=10$;

- group 2 - normal body mass mothers who delivered large for gestational age children (NBM/ LGA); $n=10$;

- group 3 - excessive body mass mothers who delivered appropriate for gestational age children (EBM/AGA); $n=10$;

- group 4 - excessive body mass mothers who delivered large for gestational age children (EBM/ LGA); $n=12$.

Leptin was determined in maternal venous blood and in umbilical cord venous blood. All blood was collected into gel separation tubes (SARSTEDT company), the maternal blood being collected before birth, and the umbilical cord blood immediately after birth. Samples were centrifuged at $1000 \mathrm{x} \mathrm{g}$, at $20^{\circ} \mathrm{C}$ for $10 \mathrm{~min}$. After centrifugation, the serum was separated, transferred into Eppendorf tubes and stored at $-20^{\circ} \mathrm{C}$. Prior to the leptin examination, samples were defrosted and mixed. Leptin was determined by the human leptin enzyme-linked immunosorbent assay (ELISA) method, using commercially available kits (Quantikine by R\&D Systems, USA). The study was performed according to the procedure specified by the manufacturer. The leptin sensitivity threshold was $7.8 \mathrm{pg} / \mathrm{ml}$, and the reference range for women = 3877-77.273 pg/ml.

\section{Statistical analysis}

Depending on the distribution of the tested variables, values are presented as medians and quartiles or as means and standard deviation. Statistical analysis was performed using Statistica 10 software (StatSoft, Cracow, Poland). The relationship between variables was determined using Pearson's correlation coefficient $(r)$. Differences between variables were examined and adjusted with one-way or two-way repeated-measures ANOVA. Respective groups were compared by ANOVA (Shapiro-Wilk test followed by Mann-Whitney $U$-test or Student $t$-test) assuming a significance level of a $p$-value less than 0.05 .

\section{Results}

In the first stage of analysis, the leptin concentration (LC) in mothers with normal body mass (NBM group) were compared to LC in mothers with excessive body mass (EBM group). A similar comparison was conducted in newborns: LC in appropriate for gestational age (AGA) children was compared with Lc in large for gestational age (LGA) children.

We found a large dispersion of LC both in maternal and newborn groups. The data are presented in Tables I and II.

In the maternal groups, mean LC in EBM mothers was found to be higher than that of NBM mothers: $32224 \mathrm{pg} / \mathrm{ml}$ vs. $14119 \mathrm{pg} / \mathrm{ml}$, $p=0.000018$ (Figure 1). Although a significant correlation was found between LC and BMI $(r=0.75, p<0.001)$, the correlations between maternal and newborn leptin concentration ( $r=$ $0.28, p=0.067)$ and between maternal LC and age $(r=0.078, p=0.622)$ were found to be insignificant.

In the newborn group, Lc was found to be higher in LGA children than AGA newborns (11789 vs. $5778 \mathrm{ml}, p=0.0084$ ) - Table II. Moderate correlations between Lc and PI ( $r=0.67, p<0.001)$, Lc and birth weight $(r=0.43, p=0.004)$ and LC and the placenta weight $(r=0.56, p<0.001)$ were observed. Despite the fact that newborn mean birth weight was similar in both sexes (girls: $3695 \pm 632$ g vs. boys: $3922 \pm 599 \mathrm{~g}, p=0.6677$ ), girls demonstrated higher mean PI $(2.1 \pm 0.24$ vs. $1.9 \pm 0.34$ $\left.\mathrm{cm} / \mathrm{g}^{3}, p=0.0125\right)$ and higher leptin median than boys: $13333 \mathrm{pg} / \mathrm{ml}$ (Q1-Q3: 7696-27976) vs. $6725 \mathrm{pg} / \mathrm{ml}((\mathrm{Q} 1-\mathrm{Q} 3:$ 5234-10 246), $p=0.0338)$ - Figure 2. It was also found that the leptin concentration in newborn serum was approximately three times lower than in maternal serum (8198 pg/ml (Q1-Q: 5639-1408) vs. 21394 pg/ml (Q1Q3: 14 645-41 301), $p<0.00002)$ - Figure 3.

In both NBM groups maternal BMI was similar and no impact of newborn nutritional status on maternal LC was observed (NBM/AGA mothers vs. NBM/LGA mothers - 12733 vs. 15144 pg/ml, 
Table I. Clinical characteristics of maternal groups

\begin{tabular}{|c|c|c|c|c|c|c|}
\hline Parameter & $\begin{array}{c}\text { NBM } \\
n=20\end{array}$ & $\begin{array}{c}\text { EBM } \\
n=22\end{array}$ & $\begin{array}{c}\text { NBM/AGA } \\
n=10\end{array}$ & $\begin{array}{c}\text { NBM/LGA } \\
n=10\end{array}$ & $\begin{array}{c}\text { EBM/AGA } \\
n=10\end{array}$ & $\begin{array}{c}\text { EBM/LGA } \\
n=12\end{array}$ \\
\hline \multirow[t]{2}{*}{ Age, mean \pm SD } & $28.29 \pm 4.97$ & $29.86 \pm 5.2$ & $27.7 \pm 5.5$ & $30.2 \pm 4.4$ & $30.1 \pm 6.4$ & $29.66 \pm 4.27$ \\
\hline & \multicolumn{2}{|c|}{$p=0.5654$} & \multicolumn{2}{|c|}{$p=0.2728$} & \multicolumn{2}{|c|}{$p=0.8514$} \\
\hline \multirow{2}{*}{$\begin{array}{l}\text { Week of delivery, } \\
\text { mean } \pm \text { SD }\end{array}$} & $39.3 \pm 1.08$ & $39.1 \pm 1.2$ & $39.1 \pm 1.2$ & $39.6 \pm 0.96$ & $38.6 \pm 1.34$ & $39.6 \pm 0.8$ \\
\hline & \multicolumn{2}{|c|}{$p=0.7542$} & \multicolumn{2}{|c|}{$p=0.3276$} & \multicolumn{2}{|c|}{$p=0.2033$} \\
\hline \multicolumn{7}{|l|}{$\%$ Body fat: } \\
\hline Median & 23 & 39.5 & 22.4 & 24.5 & 47 & 36.9 \\
\hline \multirow[t]{2}{*}{ Q1-Q3 } & $21.05-24.9$ & $36.7-46.8$ & $21.2-23.8$ & $23.4-28.8$ & $39.1-48.5$ & $35.8-39.9$ \\
\hline & \multicolumn{2}{|c|}{$p<0.0001$} & \multicolumn{2}{|c|}{$p=0.0741$} & \multicolumn{2}{|c|}{$p=0.0089$} \\
\hline \multicolumn{7}{|l|}{ \% Muscle: } \\
\hline Median & 29.3 & 26.95 & 30.25 & 27.8 & 26 & 27.3 \\
\hline \multirow[t]{2}{*}{ Q1-Q3 } & $27.8-30.9$ & $25.8-27.5$ & $29.5-31$ & $26.8-29.1$ & $25.8-27.2$ & $26.3-28.4$ \\
\hline & \multicolumn{2}{|c|}{$p<0.001$} & \multicolumn{2}{|c|}{$p=0.0986$} & \multicolumn{2}{|c|}{$p=0.0440$} \\
\hline \multicolumn{7}{|l|}{ Visceral fat: } \\
\hline Median & 5 & 8 & 5 & 5.5 & 9 & 7.5 \\
\hline \multirow[t]{2}{*}{ Q1-Q3 } & $5-6$ & $7-9$ & $5-6$ & $5-6$ & $8-12$ & $7-8$ \\
\hline & \multicolumn{2}{|c|}{$p<0.0001$} & \multicolumn{2}{|c|}{$p=0.1211$} & \multicolumn{2}{|c|}{$p=0.0023$} \\
\hline \multicolumn{7}{|l|}{$\mathrm{BMI}\left[\mathrm{kg} / \mathrm{m}^{2}\right]:$} \\
\hline Median & 22.9 & 30.9 & 20.15 & 23.65 & 32.9 & 29.9 \\
\hline \multirow[t]{2}{*}{ Q1-Q3 } & $20.1-24.2$ & $28.3-35$ & $19.5-23.7$ & $22.5-24.8$ & $31-35$ & $27.2-30.9$ \\
\hline & \multicolumn{2}{|c|}{$p<0.0001$} & \multicolumn{2}{|c|}{$p=0.0756$} & \multicolumn{2}{|c|}{$p=0.0249$} \\
\hline \multicolumn{7}{|l|}{ Newborn weight: } \\
\hline Median & 3905 & 3995 & 3070 & 4165 & 3580 & 4215 \\
\hline Q1-Q3 & $3070-4165$ & $3000-4300$ & $2890-3490$ & $4000-4370$ & $3460-3740$ & $4075-4460$ \\
\hline \multicolumn{7}{|l|}{ Leptin [pg/ml]: } \\
\hline Median & 14119 & 32244 & 12733 & 15144 & 66849 & 27620 \\
\hline Q1-Q3 & 8594-17927 & 21846-71940 & $8647-15463$ & $7865-23311$ & $30291-85295$ & $18575-36322$ \\
\hline \multirow[t]{2}{*}{ MoM, median } & 0.66 & 1.51 & 0.59 & 0.71 & 3.12 & 1.29 \\
\hline & $p=0$. & 00018 & $p=c$ & 775 & $p=c$ & 0271 \\
\hline
\end{tabular}

Table II. Clinical characteristics of newborn groups

\begin{tabular}{|c|c|c|c|c|c|c|}
\hline Parameter & AGA & LGA & AGA/NBM & AGA/EBM & LGA/NBM & LGA/EBM \\
\hline \multicolumn{7}{|c|}{ Newborn weight [g]: } \\
\hline Median & 3475 & 4180 & 3070 & 3580 & 4165 & 4215 \\
\hline \multirow[t]{2}{*}{ Q1-Q3 } & $2975-3675$ & $4040-4400$ & $2890-3490$ & $3460-3740$ & $4000-4370$ & $4075-4460$ \\
\hline & \multicolumn{2}{|c|}{$p<0.0001$} & \multicolumn{2}{|c|}{$p=0.0640$} & \multicolumn{2}{|c|}{$p=0.4682$} \\
\hline \multirow{2}{*}{$\begin{array}{l}\mathrm{Pl}, \text { mean } \pm \mathrm{SD} \\
{\left[\mathrm{cm} / \mathrm{g}^{3}\right]}\end{array}$} & $1.86 \pm 0.29$ & $2.09 \pm 0.25$ & $1.8 \pm 03$ & $1.9 \pm 0.4$ & $2 \pm 0.2$ & $2.3 \pm 0.2$ \\
\hline & \multicolumn{2}{|c|}{$p=0.0093$} & \multicolumn{2}{|c|}{$p=0.5229$} & \multicolumn{2}{|c|}{$p=0.0269$} \\
\hline \multirow{2}{*}{$\begin{array}{l}\text { Placenta weight, } \\
\text { mean } \pm \text { SD }[g]\end{array}$} & $617 \pm 78$ & $706 \pm 119$ & $605 \pm 83$ & $630 \pm 144$ & $633 \pm 89$ & $766 \pm 113$ \\
\hline & \multicolumn{2}{|c|}{$p=0.0312$} & \multicolumn{2}{|c|}{$p=0.4899$} & \multicolumn{2}{|c|}{$p=0.0101$} \\
\hline Leptin [pg/ml]: & 5778 & 11789 & & & & \\
\hline Median & $3430-11423$ & $6873-20147$ & & & & \\
\hline Q1-Q3 & 0.71 & 1.46 & & & & \\
\hline \multirow[t]{2}{*}{ MoM median } & $0.42-1.41$ & $0.85-2.49$ & $\begin{array}{c}4218 \\
3617-8034\end{array}$ & $\begin{array}{c}10293 \\
5639-13636\end{array}$ & $\begin{array}{c}6667 \\
6356-9217\end{array}$ & $\begin{array}{c}20114 \\
12022-28246\end{array}$ \\
\hline & \multicolumn{2}{|c|}{$p=0.084$} & \multicolumn{2}{|c|}{$p=0.1619$} & \multicolumn{2}{|c|}{$p=0.0013$} \\
\hline
\end{tabular}




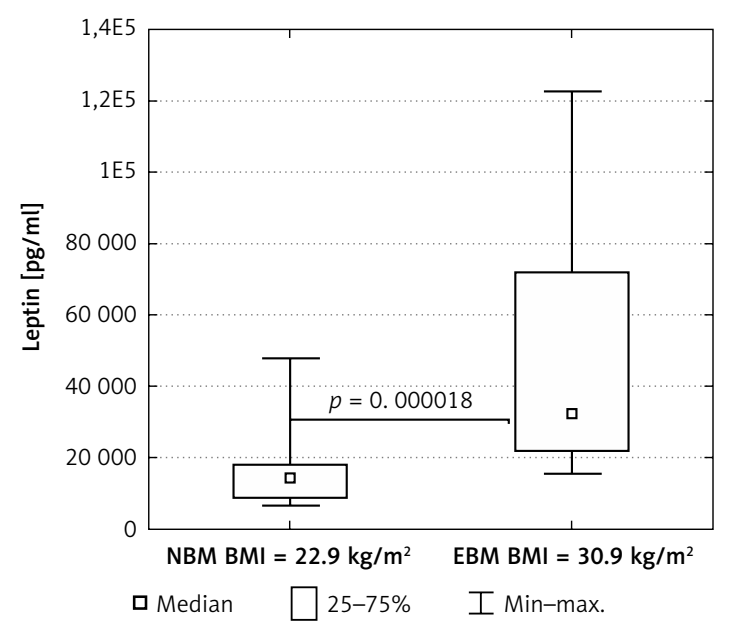

Figure 1. Comparison of maternal leptin level according to nutritional status

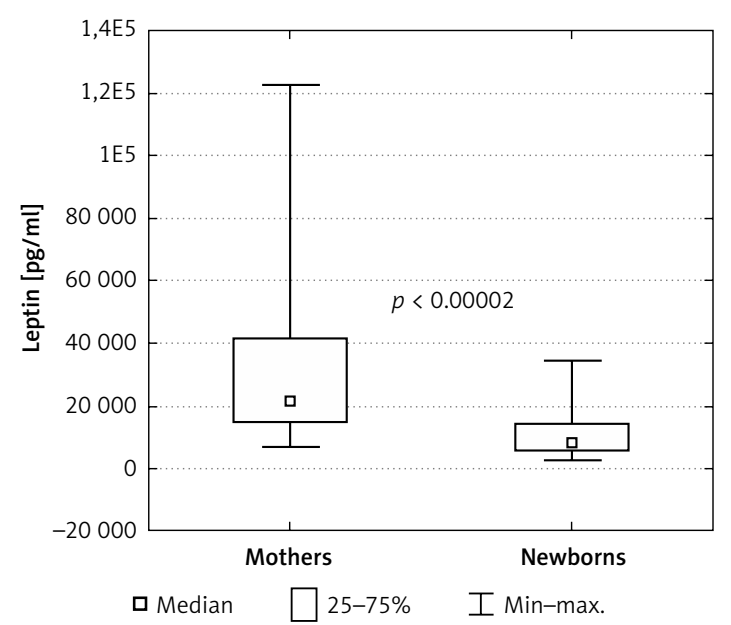

Figure 3. Comparison of leptin level in mothers and newborns groups

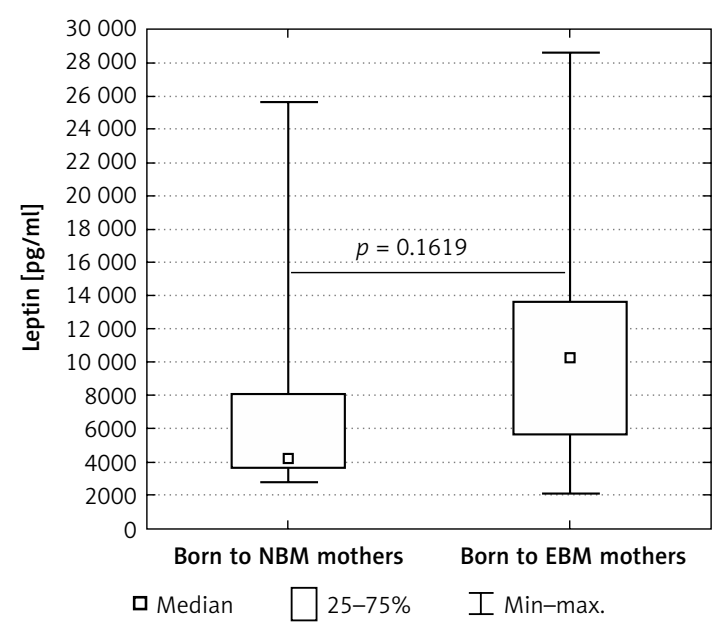

Figure 5. Leptin level in AGA newborns depending on the nutritional status of their mothers

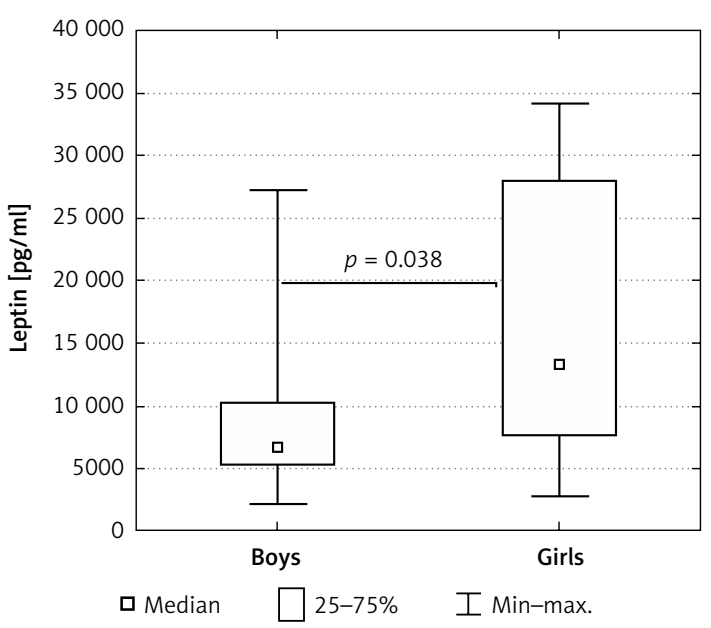

Figure 2. Leptin level in newborns in relation to gender

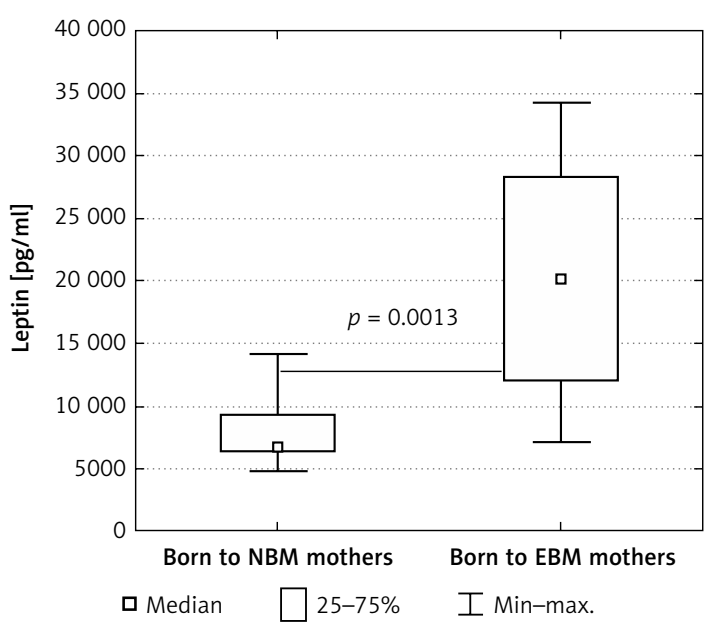

Figure 4. Leptin level in LGA newborns depending on the nutritional status of their mothers

$p=0.6775)$. A significant difference in maternal LC was found between the two EBM groups (66 $849 \mathrm{pg} / \mathrm{ml}$ in EBM/AGA mothers vs. 27620 pg/ $\mathrm{ml}$ in EBM/LGA mothers, $p=0.0271$ ). However, subgroups of EBM mothers differed in terms of BMI and body composition - Table I. Thus, the impact of newborn nutritional status on maternal LC cannot be evaluated in EBM mothers.

With regard to the impact of maternal nutritional status on newborn leptin concentration, LGA newborns born to EBM mothers presented higher LC values than LGA newborns born to NBM mothers (LGA/EBM vs. LGA/NBM newborns $=20114$ vs. 6667 pg/ml, $p=0.0013$ ) - Figure 4 . No such relationship was found in AGA children groups. Although the LC seemed to be lower in AGA/NBM newborns, there was no statistically significant difference found in median Lc (4218 pg/ $\mathrm{ml}$ for AGA/NBM vs. $10293 \mathrm{pg} / \mathrm{ml}$ for AGA/EBM, $p=0.1619)-$ Table II, Figure 5 . 


\section{Discussion}

Although the birth of an LGA newborn is usually associated with coexistent maternal gestational diabetes, the majority of LGA children are born to women with normal glucose tolerance. Currently, due to the increased incidence of obesity, elevated maternal BMI is considered to be the most important risk factor for fetal macrosomia [3, 12-16].

Correct pre-pregnancy serum leptin concentration can be interpreted as a signal that the energy resources of the mother are sufficient to make the effort associated with childbirth. This is confirmed by observations of menstrual disorders and infertility in women with a very low BMI and, consequently, very low secretion of leptin.

Maternal serum leptin concentration begins to increase in the first trimester of pregnancy, long before the weight gain associated with pregnancy becomes noticeable, which suggests that the hormone is associated with maternal metabolic adaptation to pregnancy $[2,9]$. The key role of leptin in early pregnancy may be to activate angiogenesis and to stimulate the production of extracellular matrix metalloproteinases, which modulate placental development $[2,17,18]$. However, from the beginning of the third trimester, the crucial task of leptin becomes the stimulation of maternal lipolysis in the fatty tissue [2].

In the obese pregnant woman, an excessive amount of fat stores adversely influences the proper metabolic adaptation. Obesity is associated with a chronic inflammatory process and secretion of pro-inflammatory cytokines, which leads to increased levels of triglycerides (TGs) and FFAs and, ultimately, insulin resistance [14]. The activation of lipolysis typical of normal pregnancy goes beyond the accepted limits in obese women. The increased lipolysis in the third trimester of pregnancy (stimulated by hyperleptinemia) provides the elevated concentration of glycerol necessary for maternal hepatic gluconeogenesis, as well as the FFAs used in maternal muscular oxidation processes, leaving glucose and amino acids for transplacental transport to the fetus. The placenta can transfer FFAs both by diffusion and by selective transport, increasing their concentration in fetal blood. Therefore, hypertriglyceridemia, which is accentuated by insulin resistance in obese women, is a factor that facilitates the access of nutrients to the fetus and increases fat deposition in fetal adipocytes. FFAs are known to influence glucose metabolism and to induce insulin resistance through inhibition of insulin receptor substrate 1 (IRS-1) phosphorylation, which decreases the activity of glucose transporter type 4 (GLUT4). That causes the reduction of glucose utilization by maternal tissues and facilitates glucose transfer to the fetus.
As the result of this positive feedback loop between increased lipolysis and insulin resistance in obese pregnant women, a huge prevalence of macrosomia between their newborns can be seen [3, 13, 19]. Despite this, many obese women give birth to AGA or even small for gestational age (SGA) children, which indicates the participation of other factors in fetal growth, among them the placental transport efficiency.

Leptin secreted by adipocytes is identical to the leptin produced in trophoblast cells [2]. It is therefore difficult to clearly identify whether an excessive concentration of this adipokine in maternal serum may directly impact the level of leptin in the fetal blood and to clearly identify the role of the placenta in this process.

Many authors state that the leptin concentration is higher in the mother than the newborn and these parameters do not correlate with each other $[11,19,20]$. Similar results were noted in the present study: leptin concentration was about three times higher in the blood of the mothers than the newborns. According to Hauguel-de Mouzon, this confirms that leptin does not cross the placental barrier in significant amounts [2].

It is believed that the main source of leptin in the fetus is its own adipose tissue. Numerous studies indicate a positive correlation between the serum leptin concentration in umbilical cord blood and the amount of body fat in newborns, expressed by the ponderal index (PI), and birth weight $[2,11,20]$. The findings of the present study indicate that newborn leptin level correlates better with $\mathrm{PI}$ than birth weight. Yang notes that the leptin level in the blood taken from the umbilical vein correlated more closely with maternal anthropometric parameters, while blood taken from the umbilical artery was correlated more closely with fetal biometry [16].

Placental production may be partially responsible for the elevated levels of both leptin and leptin-soluble receptors observed in pregnant women. In in vitro studies on perfused human placentas, Linnemann found that $95 \%$ of the placenta-derived leptin entered the maternal, rather than the fetal, bloodstream [21].

Since maternal leptin is not believed to enter the fetal circulation in any significant amount, its concentration in newborns may depend on the amount of their own body fat and, indirectly, on their birth weight, while the amount of maternal fatty tissue should not affect the newborn leptin concentration. However, the results of the present study indicate that Lc in LGA children born to EBM mothers was higher than in LGA children born to NBM mothers. It may be assumed that the high maternal leptin level and leptin resistance, typical for obese pregnant women, create a gradient on 
both sides of the placental barrier and enable the transplacental transport of this hormone into the fetal bloodstream. However, it is doubtful whether the large molecule leptin can cross the placenta barrier, and there are no data in the literature concerning its active transport, so other leptin-dependent mechanisms may be involved in controlling fetal growth [22].

Leptin binds to a membrane receptor, where it induces vascular endothelial growth factor receptor 2 (VEGF2) phosphorylation. Stimulated VEGF activates the $\mathrm{p} 38^{\mathrm{MAPK}}$ and $\mathrm{PI} 3 \mathrm{~K} / \mathrm{Akt}$ pathways, which increases the proliferation and mobility of cells, leading to the formation of new blood vessels [23]. Such promotion of placental angiogenesis may explain the excessive size of placentas found in many obese pregnant women. In this study the largest placentas were found in the group of LGA newborns born to EBM mothers. The increased placental leptin production may, to some degree, explain the higher level of leptin in LGA/EBM babies.

Another explanation may be that LGA children born to EBM mothers have a higher content of fat in adipocytes, as they exhibited the highest PI. The increased production of leptin by excessively fat-filled fetal adipocytes could be regarded as a defense mechanism against constant intrauterine overfeeding.

However, most interestingly, mothers from the EBM/AGA group, characterized by the highest mean leptin concentration, delivered not LGA newborns, as would have been expected, but AGA babies. According to Jansson, leptin stimulates the transplacental transport of amino acids by system A sodium-dependent neutral amino acid transport (SNAT) [18]. In addition, Farley reports SNAT impairment in obese pregnant women with hyperleptinemia compared to lean women and considers it to be the result of leptin resistance. In his work, despite the study being based on a small group of only 14 women, the author implies that the changed function of the placenta may have clinical consequences [24]. Similarly, Tessier suggests that enhanced leptin resistance due to maternal obesity may account for the limited amino acid transport to the fetus, resulting in newborn IUGR cases [8]. In the present study, babies born to mothers characterized by the highest BMI $\left(=32.9 \mathrm{~kg} / \mathrm{m}^{2}\right)$, the highest body fat content and the highest mean LC (EBM/AGA group) showed lower birth weight compared to children born to mothers with a BMI of between 25 and $32.9 \mathrm{~kg} / \mathrm{m}^{2}$.

The adverse effects of high hyperleptinemia on fetal growth may result from its action within blood vessels. Studies on the development of atherosclerotic plaques in humans demonstrate that leptin is a proinflammatory and proatherogenic cytokine. Its receptors are widely represented in both the vascular endothelium and on the plaque surface. Leptin initiates oxidative stress in endothelial cells, promotes migration and proliferation of smooth muscle cells and facilitates thrombosis by increasing platelet aggregation [25]. A similar phenomena may also occur in the vessels of the placenta, leading to their dysfunction [11].

As noted in other studies, mean Lc was found to be higher in female than male newborns, despite their similar birth weight, which suggests that sex hormones play a role in leptin secretion [2, 4].

In conclusion, leptin concentration positively correlates with $\mathrm{BMI}$ in pregnant women, with $\mathrm{PI}$ and female sex in newborns, and with placental weight. The nutritional status of fetuses does not affect the leptin level of their non-obese mothers. The LGA neonates born to women with a higher BMI demonstrate higher levels of leptin than LGA newborns born to mothers with a normal BMI, whereas leptin levels of AGA neonates are similar regardless of the nutritional status of their mothers.

\section{Conflict of interest}

The authors declare no conflict of interest.

\section{References}

1. Margetic S, Gazzola C, Pegg GC et al. Leptin: a review of its peripherial action and interactions. Int J Obes 2002; 26: 1407-33.

2. Hauguel-de Mouzon S, Lepercq J, Catalano P. The known and unknown of leptin in pregnancy. Am J Obstet Gynecol 2006: 194; 1537-45.

3. McMillen IC, Adam CL, Mühlhäusler BS. Early origins of obesity: programming the appetite regulatory system. J Physiol 2005; 565: 9-17.

4. JéquierE. Leptin signaling, adiposity, and energy balance. Ann J Y Acad Sci 2002; 967; 379-88.

5. Bouret SG. Neurodevelopmental actions of leptin. Brain Res 2010; 1350: 2-9.

6. Djiane J, Attig L. Role of leptin during perinatal metabolic programming. J Physiol Pharmacol 2008; 59: 55-63.

7. Ahima RS, Flier JS. Leptin. Annu Rev Physiol 2000; 62: 413-37.

8. Tessier DR, Ferraro ZM, Gruslin A. Role of leptin in pregnancy: consequences of maternal obesity. Placenta 2013; 34: 205-11.

9. Henson MC, Castracane VD. Leptin in pregnancy: an update. Biol Reprod 2006; 74: 218-29.

10. Monajemzadeh M, Ashtiani MT, Sadrian E, Shams S, et al. Variation in plasma leptin levels in young Iranian children with cystic fibrosis. Arch Med Sci 2013; 9: 883-7.

11. Schubring C, Kiess W, Englaro P, et al. Levels of leptin in maternal serum, amniotic fluid, and arterial and venous cord blood: relation to neonatal and placental weight. J Clin Endocrinol Metab 1997; 82: 1480-3.

12. Bloomfield FH, Spiroski AM, Harding JE. Fetal growth factors and fetal nutrition. Semin Fetal Neonatal Med 2013, Apr 30. pii: S1744-165X(13)00022-X. doi: 10.1016/j.siny.2013.03.003.

13. Heerwagen MJ, Miller MR, Barbour LA. Maternal obesity and fetal metabolic programming: a fertile epigenetic soil. Am J Physiol Regul Integr Comp Physiol 2010; 299: 711-22. 
14. Johnson AR, Milner JJ, Makowski L. The inflammation highway: metabolism accelerates inflammatory traffic in obesity. Immunol Rev 2012; 249: 218-38.

15. Hull HR, Thornton JC, Ji Y, et al. Higher infant body fat with excessive gestational weight gain in overweight women. Am J Obst Gynecol 2011; 205: 211.e1-7.

16. Yang MJ, Liu RS, Hung JH. Leptin concentrations in the umbilical vein and artery. Relationship to maternal and neonatal antropometry. J Reprod Med 2002; 47: 645-50.

17. Castellucci M, DeMatteis R, Meisser A, et al. Leptin modulates extracellular matrix molecules and metalloproteinases: possible implications for trophoblast invasion. Mol Hum Reprod 2000; 6: 951-8.

18. Jansson N, Greenwood SL, Johansson BR, et al. Leptin stimulates the activity of the system $A$ amino acid transporter in human placental villous fragments. J Clin Endocrinol Metab 2003; 88: 1205-11.

19. Higgins L, Greenwood SL, Wareing M, et al. Obesity and the placenta: a consideration of nutrient exchange mechanisms in relation aberrant fetal growth. Placenta 2011; 32: 1-7.

20. Ozdemir U, Gulturk S, Aker A, et al. Correlation between birth weight, leptin, zinc and copper levels in maternal and cord blood. J Physiol Biochem 2007; 63: 121-8.

21. Linnemann K, Malek A, Sager R, et al. Leptin production and release in the dually in vitro perfused human placenta. J Clin Endocrinol Metab 2000; 85: 4298-301.

22. Henson MC, Castracane VD. Leptin in pregnancy: an update. Biol Reprod 2006; 74: 218-29.

23. Garonna E, Botham KM, Birdsey GM, et al. Vascular endothelial growth factor receptor-2 couples cyclo-oxygenase- 2 with pro-angiogenic actions of leptin on human endothelial cells. PLoS One 2011; 6: e18823.

24. Farley DM, Choi J, Dudley DJ. Placental amino acid transport and placental leptin resistance in pregnancies complicated by maternal obesity. Placenta 2010; 31: 718-24.

25. Galkina E, Ley K. Immune and inflammatory mechanisms of atherosclerosis. Annu Rev Immunol 2009; 27 165-97. 